Results $7.12 \%$ (20/279 patients) had documented signs of anaphylaxis. Only 11 of these patients were diagnosed and treated as anaphylaxis in ED. Of the 9 unrecognised and untreated patients 4 had documented wheeze and 5 had documented airway swelling.

In addition, $2.5 \%(n=7)$ children who had no objective symptoms of anaphylaxis were treated with adrenaline (1 selfadministered, 2 paramedics, 4 in ED).

Only $11 / 20$ patients with anaphylaxis were referred to paediatrics for observation and allergy assessment.

In $70 \%$ cases of anaphylaxis $(n=14)$, the trigger was food. In $30 \%(n=6)$ no trigger was identified. Tree nuts $(42 \%, 6$ cases $)$ was the most common food trigger for anaphylaxis.

While allergic reactions are more common in younger children (228 under 10 years vs 51 cases $11-16$ years.), the proportion presenting in anaphylaxis is much higher in adolescents with $22 \%$ (11/51) presenting in anaphylaxis vs 4\% (9/219) in 010 year olds.

Conclusion Our audit has given insight in to the age, sex, triggers and symptoms of children presenting in anaphylaxis and identifies disparity in the recognition and management of anaphylaxis in the ED. To standardise and improve care, it was recommended that a paediatric anaphylaxis care pathway should be implemented in line with the current guidance.

\section{G63(P) A COMPARITIVE ANALYSIS OF CONCURRENT MALARIA AND DENGUE INFECTION WITH EITHER INFECTION ALONE}

A Ahmad, IA Khan. Paediatrics, JN Medical College and Hospital, Aligarh Muslim University, Aligarh, India

\subsection{6/archdischild-2018-rcpch.61}

Aim In tropics, malaria and dengue are endemic and co-infections are common and often misdiagnosed as monoinfection. Only few co-infection cases have been reported in children, with insufficient data to enhance the understanding of the effects of co-infection. This study compared the clinico-haematological profile of malaria and dengue mono-infection with co-infection.

Methodology This was a cross-sectional observational study of patients hospitalised with acute febrile syndrome (history of fever in the past 7 days) who were investigated for malaria, dengue and other causes of fever. Clinical features and haematological parameters of malaria, dengue were compared with co-infections.

The diagnosis of malaria was confirmed by thick and thin blood smear microscopy. The diagnosis of dengue was made either by a positive serology (IgM) or a positive NS1 antigen or a positive molecular test (RT-PCR), considering that every patient was tested by all the three methods. Severity was classified and managed according to the World Health Organisation (WHO) guidelines for dengue and malaria

Results One hundred and thirty nine children positive for dengue, malaria or both, were analysed. Fifty-two (37.4\%) were malaria, 74 (53.2\%) were dengue and 13 (9.3\%) were co-infection. Patients with co-infection had more chills and pruritis as compared to malaria and dengue $(p<0.05)$. Bleeding manifestations like petechiae were comparable in both coinfection and dengue but not observed in malaria. Neurological manifestations like altered sensorium and seizures were not observed in dengue $(\mathrm{p}<0.05)$ but comparable in malaria and co-infections. Jaundice was observed only in malaria. Hepatomegaly was comparable in all groups whereas splenomegaly was noted more in co-infections. Anaemia was observed significantly more in malaria $(\mathrm{p}<0.05)$ while other parameters like WBC, platelets and renal functions were comparable.

Conclusions A high index of suspicion for co-infection should be suspected in endemic areas for malaria and dengue. Jaundice and anaemia (in dengue patients) and spontaneous bleeding (in malaria patients) should raise the suspicion of coinfection. Whenever co-infection is confirmed, we recommend careful monitoring for bleeding and hepatic complications, which may result in a higher chance of severity, despite of the fact that no increased fatality rate was seen in our group.

\section{G64(P) AN ASSESSMENT OF THE DIAGNOSIS AND TREATMENT OF CYTOMEGALOVIRUS AT THE UNIVERSITY HOSPITAL OVER THE LAST 10 YEARS}

E O'Loan, C Jones. Faculty of Medicine, University of Southampton, Southampton, UK

\subsection{6/archdischild-2018-rcpch.62}

Background and aims Congenital cytomegalovirus (cCMV) is the most common congenital infection with a birth prevalence of $0.3 \%-0.7 \%$ in developing countries. It is the leading cause of non-genetic sensorineural hearing loss (SNHL) and an important cause of neurodisability. At birth, 10\%-15\% of infants are symptomatic and a further 10\%-15\% develop symptoms, mostly SNHL, by age 5 years. Despite this, there is limited awareness of cCMV, therefore meaning many infected infants are undiagnosed.

We aimed to determine the number of infants presenting to our University Hospital with cCMV over a 10 year period and to describe the diagnosis, treatment and outcome of these infants in order to refine our clinical pathways.

Methods Electronic patient databases were searched to identify patients diagnosed with cCMV between 01/09/2007 and 30/ 08/2017. A standardised data capture tool was designed and utilised to describe the clinical features and management of these children.

Results Forty infants were initially identified of which 4 were subsequently determined to have postnatal acquisition of CMV. Of the 36 infants with cCMV, 22 (61\%) were symptomatic in the neonatal period and $6(17 \%)$ developed sequelae beyond the neonatal period. The mean age of diagnosis was 4 months 10 days. The majority of infants were diagnosed using PCR of a urine sample. Eighteen (50\%) of infants were treated with either ganciclovir or valganciclovir for either 6 weeks or 6 months. In total, $28(78 \%)$ infants experienced long term adverse outcomes associated with CMV.

Conclusion Congenital CMV is a relatively rare condition, however it is associated with a significant health burden for infants, their families and the NHS. Increased awareness and education of pregnant women and healthcare professionals is essential to improve early diagnosis of cCMV and therefore increase the number of infants who might benefit from antiviral treatment. Follow-up of both symptomatic and asymptomatic infants is essential for early diagnosis of SNHL and therefore early intervention for these infants. 